\title{
Philosophiques
}

\section{L'attention et le souci des autres dans un monde indifférent : geste moral ou conditionnement social ? À propos de $L a$ fragilité du souci des autres. Adorno et le care, par Estelle} Ferrarese

\section{Pascale Devette}

Volume 46, numéro 1, printemps 2019

URI : https://id.erudit.org/iderudit/1062020ar

DOI : https://doi.org/10.7202/1062020ar

Aller au sommaire du numéro

Éditeur(s)

Société de philosophie du Québec

ISSN

0316-2923 (imprimé)

1492-1391 (numérique)

Découvrir la revue

Citer cet article

Devette, P. (2019). L'attention et le souci des autres dans un monde indifférent : geste moral ou conditionnement social ? À propos de La fragilité du souci des autres. Adorno et le care, par Estelle Ferrarese. Philosophiques, 46(1), 229-242. https://doi.org/10.7202/1062020ar d'utilisation que vous pouvez consulter en ligne. 


\section{Étude critique}

L'attention et le souci des autres dans un monde indifférent: geste moral ou conditionnement social? À propos de La fragilité du souci des autres. Adorno et le care, par Estelle Ferrarese'

\section{PASCALE DEVETTE}

Université de Montréal

L'enjeu général du livre La fragilité $d u$ souci des autres s'inscrit dans une réflexion sur la compartimentation sociale du soin et de l'attention aux autres. Estelle Ferrarese précise ses analyses au travers d'un dialogue jusquelà inédit entre Theodor W. Adorno, les éthiques du care ainsi que la sensibilité commune qu'on retrouve dans ces deux corpus concernant le souci des autres.

En partant de l'importance des besoins, les théoriciennes et les théoriciens du care se positionnent contre un pan majeur de la philosophie politique moderne qui intègre le geste moral dans une éthique universelle et abstraite (largement inspirée de Kant). Les théoriciennes du care affirment la primauté des besoins sur les droits. Bien évidemment, elles ne rejettent pas la portée des droits, mais considèrent que les droits naissent des besoins, dont il faut être à l'écoute. Les besoins ne peuvent pas tous être prédéfinis, il faut donc être sensible aux situations diverses. Ainsi, plutôt que de demander "qu'est-ce que la justice?", les théoriciennes du care ancrent leurs interrogations dans des situations concrètes et cherchent à circonscrire les besoins d'une personne et à y répondre le mieux possible. Ce faisant, cette approche de l'éthique permet aussi de jeter un regard neuf sur la vulnérabilité. La possibilité d'être affecté par le monde et l'interdépendance qui nous lie les uns aux autres ne sont pas considérées comme des tares; au contraire, ces éléments soutiennent une anthropologie de la vulnérabilité en tant que lien social majeur et central. En effet, le quotidien est constitué de petits gestes de soin, d'attention et d'écoute qui rendent le monde vivable. Les êtres humains sont des êtres relationnels, bien que par ailleurs, les personnes qui prennent davantage soin des autres soient souvent moins remarquées, comme si ces gestes allaient de soi ou avaient peu d'intérêt.

Les éthiques du care ont donc pour objectif de souligner l'importance, dans nos gestes moraux, du particulier et de la souffrance (toujours située) de chaque personne en tant qu'être vulnérable. Elles fondent leurs approches sur l'importance de l'écoute, de l'attention et de l'observation: il s'agit d'être réceptif à son environnement afin d'agir pour répondre à un besoin de la

1. Estelle Ferrarese, La fragilité du souci des autres, Lyon, ENS Éditions, 20 I 8, I 50 pages. 
manière la plus adéquate. Ferrarese suit cette voie tracée par les éthiciennes du care et définit

l'attention comme attitude adéquate[, donc] par définition un geste ou une façon de faire (ou de ne pas faire) ajustés aux besoins du destinataire, fussentils de distance ou de détachement, et accordés car survenant au bon moment. Elle suppose une sensibilité aux états émotionnels de la personne dont il s'agit de prendre soin, elle nécessite une attitude expérimentale, ou "délicatesse", ou encore la capacité à passer à autre chose dans certaines situations².

Mais si l'attention au particulier est nécessaire, ce n'est pas seulement en ce qu'elle permet de répondre adéquatement aux besoins du destinataire. En donnant une visibilité théorique aux gestes de soin, les théoriciennes du care cherchent également à politiser et à faire reconnaître l'importance sociale du travail de soin et des personnes, souvent discrètes ou maintenues dans l'ombre, qui veillent à la pérennité du monde et des autres. Ainsi, Joan Tronto considère que la reconnaissance du care, sur le plan des valeurs tant individuelles qu'institutionnelles, va de pair avec une amélioration de nos capacités d'attention aux autres, au sens où l'attention n'est pas simplement une posture individuelle dont certaines sensibilités seraient porteuses, mais un fait social qui peut être encouragé et dont on peut faire l'apprentissage ${ }^{3}$. Marie Garrau va également dans ce sens lorsqu'elle nous convie à l'importance de «rendre le monde attentif ${ }^{4}$ ».

Il est vrai que la pensée d'Adorno pourrait, dans un premier temps, sembler éloignée des éthiques du care, notamment lorsqu'on considère les propos d'Adorno sur les femmes. Ferrarese relève quelques affirmations tenues dans La dialectique de la raison par Horkheimer et Adorno en ce qui concerne le rôle des femmes. Pour les deux auteurs, les femmes

ne prennent pas une part personnelle à l'activité efficace sur laquelle est basée cette civilisation. L'homme doit sortir dans le monde hostile, c'est à lui d'agir et de lutter. La femme n'est pas un sujet. Elle ne produit rien, mais se contente de s'occuper de ceux qui produisent ${ }^{5}$.

Il semble ici qu'Adorno et Horkheimer dessinent une vision de la femme naturellement portée vers la passivité et l'objectivation, à l'opposé de l'homme qui serait actif et sujet. Un chapitre de La fragilité du souci des

2. Ibid., p. 24 .

3. Joan Tronto, Moral Boundaries: A Political Argument for an Ethic of Care, London, Routledge, I993.

4. Marie Garrau, "Le care est-il soluble dans la non-domination?", dans Garrau et Le Goff (dir.), Politiser le care? Perspectives sociologiques et philosophiques, Lormont, Le Bord de l'eau, p. I I6.

5. Max Horkheimer, Theodor W. Adorno, La Dialectique de la Raison, Paris, Gallimard, coll. «Tel», I974, p. 270. Cité par Estelle Ferrarese, «(Re)faire de la Théorie critique. Pour une (re)lecture féministe de l'École de Francfort ", Cités, n 73, 20I8/I, p. 44. 
autres est d'ailleurs consacré à « un oubli dans la thèse de l'oubli », et cherche à corriger la théorie adornienne de l'indifférence, c'est-à-dire l'idée selon laquelle les relations sociales seraient marquées par l'oubli de la sensibilité pour les autres, par les approches féministes du "care", plus particulièrement sur la distribution genrée de l'attention aux autres. Sous bien des aspects, les femmes sont perçues dans «l'œuvre d'Adorno comme les objets de déterminations caricaturales, qui les assignent à des figures pathétiques et obligatoires, «la » mégère, «la» jeune fille, «la » balayère, «l' »épouse, etc., qui ensemble composent «la» femme ${ }^{6} »$. Il y a, en effet, dans la théorie critique de l'École de Francfort, peu de sensibilité féministe.

Cet aspect n'est pas propre aux anciennes générations de la théorie critique, puisque même chez les représentants plus récents, notamment Harmut Rosa, on peut être surpris par le peu de cas qui est fait de la condition féminine, d'une part, et par la moindre prise en considération des aspects tout à fait pertinents des théories féministes, d'autre part. Rosa, dans ses recherches sur l'accélération, examine peu la dimension temporelle des femmes en tant que catégorie de la population encore largement chargée de prendre soin des autres. Pourtant, les études sur la «charge mentale» des femmes, sur la «double journée de travail» et sur les difficultés inhérentes au fait de devoir jongler entre le temps du corps (les corps des nourrissons, des malades, des personnes âgées) et le temps abstrait (rationalisé par l'horloge, donc désincarné de la matérialité des choses) sont tout à fait liées à la problématique de l'accélération des modes de vie dans la modernité tardive ${ }^{7}$. Rosa lui-même admet qu'il aurait pu aborder l'aspect genré de la dimension sociale du temps, mais cet élément, selon lui, est «secondaire» dans sa démarche, qui consiste plus largement à saisir la modernité tardive à partir de l'accélération en tant que phénomène totalisant ${ }^{8}$. En ce sens, la question du genre semble perçue comme une niche précise, ne concernant qu'une

6. E. Ferrarese, La fragilité du souci des autres, op. cit., p. 8I.

7. Pensons, bien entendu à la littérature des éthiques du care (voir Laugier, Sandra, Paperman, Patricia, Le souci des autres. Éthique et politique du care. Paris, Éditions de l'École des hautes études en sciences sociales; Evelyn Nakano Glenn, "Creating a Caring Society", Contemporary Sociology, 29, I, 2000, p. 84-94 ou, encore, Nel Noddings, Starting at Home. Caring and Social Policy, Berkeley, University of California Press, 2002), mais aussi à plusieurs recherches en sociologie du travail, par exemple les écrits de Dominique Méda (Le temps des femmes: pour un nouveau partage des rôles sociaux, Paris, Flammarion, 2008). À l'instar de Hartmut Rosa, plusieurs auteurs et autrices se penchent précisément sur la question de la temporalité, mais à la différence de ce dernier, ils prennent en considération la manière dont l'accélération du temps influence différemment les catégories de la population. Notons par exemple les travaux de Juliette Schor (Plenitude: The New Economics of True Wealth, Penguin Press, 20I0) et de Jonathan Martineau (L'ère du temps, modernité capitaliste et aliénation temporelle, Montréal, Lux Editeur, 2017).

8. Hartmut Rosa, Accélération. Une critique sociale du temps, Paris, La Découverte, 20IO, p. 24-25. 
partie de la population, tandis que la théorie critique de l'École de Francfort serait, elle, mue par une ambition holistique et générale.

Dans ce contexte, le livre de Ferrarese s'avère judicieux et salutaire, car il propose des ponts entre les deux approches, mais il le fait sans complaisance, en prenant soin de complexifier et de montrer certaines limites propres à l'un et l'autre des deux corpus. L'exercice ne consiste pas seulement à établir des comparaisons, il permet de désenclaver les littératures afin de réfléchir plus en profondeur sur le souci des autres dans un monde structuré par l'indifférence. Ferrarese y approfondit ses convictions développées ailleurs et montre combien la théorie féministe a des choses à dire sur le général ${ }^{9}$. L'éclairage conjoint de la pensée d'Adorno et des éthiciennes du care sur le souci des autres introduit une dimension structurelle et systémique à la notion d'attention, encore trop peu exprimée dans la théorie politique. Ce faisant, les femmes et les subalternes, en tant que catégorie sociale, ne sont plus considérées uniquement dans leurs particularismes, ou encore, la question du souci des autres n'est plus uniquement reléguée aux approches féministes. En fait, Ferrarese révèle la pertinence de ces éléments (les donatrices de soin et les théories féministes du care) en les posant au cœur des problématiques du capitalisme, plus précisément en donnant à ces questions une force générale telle qu'inspirée par l'École de Francfort. Ferrarese réussit en ce sens très bien à révéler l'importance du souci des autres pour toutes et tous, à tous les niveaux du social et du politique.

L'autrice suit, ce faisant, une ligne directrice dans laquelle elle prend soin de montrer les points d'ancrage propres à la théorie d'Adorno et aux éthiques du care. Dans les deux cas, une place prépondérante est accordée au corps, à la souffrance et à la pratique dans sa relation au geste moral. Les deux corpus se rejoignent également dans l'attention portée envers le particulier et la singularité de la personne, et s'opposent à une certaine forme d'homogénéisation créée par un mauvais usage de l'universel. On retrouve également chez Adorno et chez les éthiciennes du care une manière d'envisager le geste moral dans son contexte, dans sa particularité, dans son émotivité, posant ainsi la morale en situation. Finalement, dans les deux cas, on peut réfléchir à une forme de relationalité du soi qui est déjà imbriqué dans le monde, déconstruisant par-là l'idée d'une nature interne au sujet qui serait imperméable à l'extériorité sociale historique du monde.

9. Dans «(Re)faire de la théorie critique. Pour une (re)lecture féministe de l'École de Francfort " (Cités, $\mathrm{N}^{\circ} 73,20 \mathrm{I} 8 / \mathrm{I}$ ), Ferrarese écrit : «nous en conserverons une posture générale, qui tient à la conviction que le féminisme a quelque chose à dire sur la participation, sur la réification, sur le capitalisme, sur la crise, ou encore sur les processus de subjectivation en général. La Théorie critique féministe refuse de déserter le général parce que les femmes en ont été chassées. Elle est portée par la conviction que le féminisme peut et doit modeler le geste critique, que celui-ci s'exerce ou non sur le patriarcat et la domination masculine » (p. 45). 


\section{L'empire de la froideur}

Au premier abord, on pourrait se questionner sur la pertinence de revenir à Adorno. En effet, maintes théoriciennes du care ont réfléchi à l'aspect politique inhérent à la distribution du soin. Ainsi, Joan Tronto a abordé plusieurs éléments qui détournent ou entravent l'attention aux autres, notamment le phénomène de surinformation et la tendance qui y est liée à écarter davantage les informations de détresse que les informations de plaisir. Cette propension est exacerbée par la société de consommation. En effet, la personne en moyen financier peut être amenée à moins ressentir sa propre dépendance aux autres, comme elle peut plus difficilement percevoir sa responsabilité envers ceux et celles qui l'entourent.

Or c'est précisément sur ce sujet, soit le phénomène de l'indifférence, qu'Adorno s'est penché afin de comprendre l'avènement de «L'Empire de la froideur». Inspiré par l'archétype de la subjectivité bourgeoise, Adorno conçoit le règne de la froideur comme un ensemble de relations sociales non relationnelles. Ce type d'interactions fonctionnent grâce à l'absence de lien véritable entre les sujets. La froideur est régie par l'indifférence envers les autres, par la capacité à ne pas être touché par eux, à ne pas s'identifier à autrui, elle encourage la compétition avec l'ensemble des êtres humains et incite l'uniformisation des comportements de manière à ce qu'ils incarnent la norme des dominants.

À l'opposé, donc, des éthiciennes du care qui examinent la question de la sollicitude (bien qu'elles réfléchissent à la compartimentation des soins), Adorno part du constat de l'absence de souci pour les autres et réfléchit à la structuration d'un monde qui encourage l'insensibilité. On voit chez lui se dessiner une conception très individualiste de la personne, bien que ce principe d'identité ne soit en rien subjectivant: en réalité, le sujet perd de son expérience du monde. Pour le dire simplement, la singularité froide d'Adorno croit penser à elle lorsqu'elle s'identifie et travaille à devenir un individu autonome et productif, alors que dans les faits, elle s'assujettit au processus d'abstraction universel propre au capitalisme. Cet assujettissement est concomitant à la conception du sujet rationnel kantien. La raison, pour Adorno, vise l'établissement d'un savoir comme mode de domination de la nature par la technique ${ }^{10}$. La moralité n'est ainsi jamais détachée de son contexte d'apparition. La froideur participe à la fois des pratiques sociales, des institutions, de l'industrie culturelle, comme des perceptions et des expériences subjectives.

10. La domination de la nature en tant que processus civilisationnel d'instrumentalisation du monde a été développée dans La dialectique de la Raison. La nature, note Ferrarese, réfère à certains corps plus socialement conçus comme naturels "celui des juifs, celui des femmes [.] La nature, perçue comme faiblesse et fragilité, suscite une pulsion sadique. Et les êtres rationnels que nous sommes tous devenus ne voient guère la raison d'une sollicitude pour l'animal privé de raison" (La fragilité $d u$ souci des autres, p. 33-34). 
Partant de ce monde faux, Ferrarese examine la critique adornienne de l'immédiateté de l'expérience subjective. En fait, l'expérience ne fonde plus une connaissance juste permettant de guider le rapport que nous entretenons avec les autres, puisque cette expérience est déjà gangrénée par un ensemble de mutations qui chosifient les relations à soi-même, au monde et aux autres. Adorno observe, dans différents processus, dynamiques sociales, culturelles et institutionnelles ${ }^{11}$, un phénomène de diminution de la capacité à percevoir réellement quelqu'un. La structure du monde administré nous contraint à «oublier» autrui. De fait,

les rencontres avec autrui ne sont plus, dans les conditions d'une expérience atrophiée, que de simples entrechoquements. [L]e lien de l'indifférence à autrui à la généralisation de l'expérience brute procède aussi de ce que, loin d'individualiser le sujet face à autrui, de lui conférer son unicité, l'expérience atrophiée du capitalisme tardif devient interchangeable et standardisée ${ }^{12}$.

L'échange qui structure le lien social se construit sur la négation de la singularité afin de façonner des manières de concevoir autrui comme être remplaçable et instrumentalisable. Cet oubli de l'autre est concomitant à l'atrophie de l'expérience vécue et s'arrime à des formes de relations dans lesquelles chacun et chacune deviennent commensurables, calculables.

Si l'empire de la froideur se diffuse sur les relations sociales en les quantifiant au gré des échanges et des profits, la subjectivité froide est construite par l'impératif de "la conservation de soi », forme la plus aliénée de socialisation. C'est grâce au formatage des idéaux portés par la rationalité instrumentale et l'autonomie égocentrique, intégrés par la subjectivité froide, que cette dernière arrive à incarner la conservation de soi, en tant qu'indifférence pour les autres. Comme l'explique Ferrarese: «la philosophie morale moderne a fait de l'indifférence mutuelle la condition de la morale et de la justice; l'idée de norme morale est construite sur le présupposé d'une absence d'intérêt pour autrui ${ }^{13}{ }^{»}$. En ce sens, une théorie morale qui propose un modèle abstrait qu'il suffirait d'appliquer contribue précisément à l'oubli du particulier en y plaquant l'universel. Il faut donc, suivant Adorno, rendre sa dignité au singulier, ce qui ne veut pas dire renier toutes formes d'universalités. Pour lui, la connaissance morale implique un regard sur les contradictions et les paradoxes qui habitent le singulier dans son

11. Par exemple, Ferrarese revient sur la critique adornienne de l'industrie culturelle, sur l'organisation du travail en usine, sur la bureaucratie, sur le langage. Dans tous ces exemples, il y a substitution de l'expérience vécue par la répétition du même, qu'il s'agisse de répétition de slogan, répétition de gestes mécaniques ou répétition de règlements. Ces différents éléments tendent à dissoudre l'expérience vraie et à la transformer par des séquences sans signification.

12. Ibid, p. 47-48.

13. Ibid., p. 58 . 
rapport tendu à l'universel. Ces contradictions révèleraient les exigences imposées par une certaine situation sociale historique.

À ce titre, Ferrarese rappelle que la connaissance morale adornienne fait une place à la spontanéité, comprise comme une réaction impulsive qui va à l'encontre de l'impératif de la conservation de soi. Par exemple, Adorno considère que l'être humain aurait une aversion contre la souffrance: l'impulsion à soulager la souffrance serait une émotion "pré-égoïque » ${ }^{14}$. Ferrarese explicite ici avec justesse le matérialisme critique d'Adorno. L'impulsion qui provoque l'émotion et guide le possible geste moral n'est pas intentionnelle, car la personne ne choisit pas par quoi elle est affectée:

Le sursaut de dégoût devant la torture infligée à un autre corps n'est pas identification à un moi qui me ressemble, il ne repose pas sur une prise de rôle ou sur une orientation vers les raisons d'autrui, ni ne les suscite. Il ne rapproche que deux corps. [...] l'objectivité se rappelle ainsi, par une pression sur l'organisme, aux impulsions et à la conscience du sujet ${ }^{15}$.

La reprise adornienne orchestrée par Ferrarese rejoint ainsi de plusieurs manières la lecture développée par Judith Butler d'Emmanuel Levinas dans Le récit de soi ${ }^{16}$. La vulnérabilité du visage de l'autre, nous dit Butler, est une forme d'interpellation pré-individuelle qui contredit l'idée même de préservation (conservation) de soi : être affecté par autrui et se sentir responsable moralement implique une affectation de soi qui modifie l'individualité. Pour Butler, il importe

de considérer, comme Lévinas, que la préservation de soi n'est pas le but le plus élevé, ni la défense d'un point de vue narcissique le besoin psychologique le plus pressant. Le fait que nous soyons sous emprise à l'encontre de notre volonté et de manière primaire est le signe de notre vulnérabilité et d'une dette vis-à-vis d'autrui, auxquelles nous ne pouvons pas échapper. Nous ne pouvons nous en défendre qu'en soulignant le caractère asocial du sujet au détriment d'une relationalité difficile et intraitable, parfois même insoutenable ${ }^{17}$.

Le règne de la froideur tend justement à nier la vulnérabilité commune, à diminuer l'impulsion devant la souffrance en y apposant une conception asociale de l'être humain (comme individu visant sa conservation) de manière à compliquer, voire rendre impossible, l'actualisation du souci des autres. La question n'est pas, chez Adorno, de retrouver une première nature sensible à la souffrance qu'une seconde nature camouflerait, mais bien de cibler ce qui, structurellement, contribue à empêcher la perception (pourtant matérielle, car elle implique le corps) de la souffrance. En effet, la froideur se déploie grâce à l'oubli de ce qui est pourtant perçu.

14. Ibid., p. I7.

15. Ibid., p. I8.

16. Judith Butler, Le récit de soi, Paris, Presses Universitaires de France, 2007.

17. Ibid., p. I02. 
Ainsi, Ferrarese avance qu'il lui "paraît possible de soutenir que, de même, la perception, qui a bien lieu, de tous les aspects d'un objet, de tous les possibles d'une réalité, de la détresse d'autrui, est frappée de non-pertinence, et avec elle, l'horizon d'obligations, responsabilités et doutes qui se déploierait si nous n'oubliions pas cette perception ${ }^{18}{ }^{\prime}$. La valeur intrinsèque de la perception de la souffrance est, par l'oubli même de cette perception, neutralisée, reléguée parmi ce qui ne compte et n'appelle, donc, aucun geste moral.

\section{L'oubli dans la thèse de l'oubli}

Tout au long de son analyse, Ferrarese montre bien comment le capitalisme fonctionne en compartimentant l'attention, notamment en assignant les tâches de soin à certaines personnes, surtout aux femmes et aux subalternes. C'est ici que se loge l'oubli d'Adorno, qui n'interroge pas la signification sociale de l'assignation catégorielle du souci des autres. À cet égard, Ferrarese prend soin d'actualiser les transformations néolibérales depuis l'époque d'Adorno. Elle constate par exemple une libération de l'émotivité, qui ne doit pas être confondue avec une liberté des émotions ${ }^{19}$. Au contraire, le néolibéralisme provoquerait une stimulation de certaines émotions précises déjà reconnaissables, encouragées et orientées vers des catégories précises. C'est tout l'aspect de l'auto-entrepreneur qui sait être émotionnel en tant que l'émotion fait partie de l'échange dans une forme de relation non relationnelle aux autres, au sens où chacun est commensurable, et où l'expérience n'est pas singulière mais répétable, comme l'est un exemplaire.

La démarche de Ferrarese fait alors écho en partie aux travaux de Nancy Fraser qui reprend ceux de Luc Boltanski et d'Ève Chiapello dans Le Nouvel esprit $d u$ capitalisme ${ }^{20}$. Ainsi, le capitalisme tardif s'organise en partie grâce à la neutralisation de la critique féministe de la deuxième vague à travers un déplacement de l'assignation aux soins. Au fur et à mesure que les femmes occidentales prennent place dans le marché du travail, qu'elles acquièrent une visibilité et certains privilèges, l'économie des soins se voit graduellement déférer à d'autres catégories de la population, toujours précaires et pratiquement invisibles ${ }^{21}$. Chez les théoriciennes du care, ce phénomène est expliqué par «l'irresponsabilité du privilégié" ou "l'indifférence

18. E. Ferrarese, La fragilité du souci des autres, op. cit., p. 76 .

19. Voir notamment les travaux d'Eva Illouz, Saving the Mordern Soul. Therapy, Emotions, and the Culture of Self-Help, Berkeley, University of California Press, 2008.

20. On peut penser à l'article suivant: Nancy Fraser, «Feminism, Capitalism and the Cunning of History ", New Left Review, ${ }^{\circ}$ 56, March/April 2009, p. 97-I I 7 .

21. Sur ce point, les travaux de Caroline Ibos sur les «nounous africaines» et leur relation non relationnelle avec les femmes qui les emploient sont révélateurs des phénomènes tout à fait actuels de chosification de l'humain (Caroline Ibos, "Du macrocosme au microcosme, du vaste monde à l'appartement parisien, la vie morale de la Nounou ", Multitudes, n 37-38, 2009, p. I23-I3I). 
du privilégié $»^{22}$. La confrontation entre la thèse de l'oubli d'Adorno et les éthiciennes du care permet à Ferrarese de constater que la fragilité du souci des autres ne relève pas d'une sensibilité morale, mais plutôt d'une distribution sociale des rôles de soin. Ainsi, l'autrice avance

l'idée de subjectivations différenciées en fonction du genre[.] L'enjeu est bien d'envisager comment au sein d'une forme de vie qui institue une indifférence généralisée, une attention à autrui compartimentée, limitée à des modes et à des domaines susceptibles de produire du travail ou une consommation particulière, est impartie aux femmes ${ }^{23}$.

En ce sens, l'objectif n'est pas de glorifier une subjectivité typiquement féminine admirable contre une subjectivité masculine aliénée, mais de saisir le mécanisme de compartimentation de l'attention en tant que résultant d'une structure d'indifférence aux autres. L'empire de la froideur d'Adorno prend donc un aspect encore plus global à travers les liens qui sont tissés avec les éthiques du care, puisque l'indifférence généralisée implique que certaines catégories de personnes soient disposées moralement à s'occuper des autres. Afin de pouvoir traiter les êtres humains comme des objets, il est nécessaire qu'une partie de la population soit dévolue à l'entretien du lien humain, des corps, de l'environnement au sens large. Plutôt qu'une diffusion généralisée de la capacité à se soucier d'autrui, on voit ici apparaître une spécialisation des gestes moraux imposés à certains groupes dont on approprie le travail et par laquelle la pérennité de l'indifférence globale est possible.

\section{Le sens de l'attention et du souci des autres}

L'attention envers les autres et envers l'environnement s'inscrit davantage dans une socialisation tout à fait cohérente et nécessaire au soubassement de l'efficacité contemporaine: le care résulte ici d'une économie de la commensurabilité généralisée. Le savoir moral, dont nous avons souligné la critique rationaliste, est de plus en plus difficile à cerner en situation, notamment par suite du phénomène d'accélération du flux d'informations et de choses à faire. Le discernement du geste approprié est également complexifié par les multiples conséquences possibles de ce geste ${ }^{24}$. La question, dès lors, revient à demander "ce qu'il reste, de ce qu'il peut rester de contenu moral, de justesse dans des actes de care résultant de l'économie générale des affects

22. E. Ferrarese, La fragilité du souci des autres, op. cit., p. 98.

23. Ibid., p. 99-I00.

24. Ferrarese renvoie à l'exemple illustré par Michel Callon (dans son article «La leçon d'humanité de Gino ", Réseaux, no 95, I999, p. I97-233) pour aborder la densification contemporaine de nos choix moraux. Dans le même ordre d'idée, la discussion du concept d'action telle qu'élaborée par Hannah Arendt dans La condition de l'homme moderne aurait été tout à fait pertinente. 
caractéristiques de la forme de vie capitaliste ${ }^{25}$ ». Le souci des autres, en tant qu'il a été historiquement assigné à un groupe (particulièrement les femmes) et qu'il résulte d'une différenciation rationalisable des tâches de soin, est-il faux et simplement le résultat d'un système mauvais ?

L'autrice ne répond pas clairement à cette problématique, qui me semble être un aspect irrésolu du livre, mais le fait de ne pas trancher la question apporte plus de substance à la réflexion qu'il n'en enlève. Dans une certaine mesure, on pourrait avoir l'impression que Ferrarese rejoint la thèse défendue par Elsa Dorlin dans Se défendre. Une philosophie de la violence ${ }^{26}$. Dorlin analyse plusieurs systèmes de domination (en situation coloniale, dans le ghetto de juif de Varsovie, dans les mouvements noirs aux ÉtatsUnis) avec une perspective foucaldienne, en réfléchissant aux pratiques de résistances au pouvoir dans des situations d'impuissance radicale. En considérant la construction mutuelle du pouvoir et du sujet, Dorlin soutient que la disposition à être attentif à l'autre relève en partie d'une "phénoménologie de la proie». La précarité et la violence structurelle, voire corporelle, dont sont victimes les catégories sociales assignées aux tâches de soin façonnent, et même conditionnent, l'attention aux autres. Pour Dorlin,

la violence endurée génère une posture cognitive et émotionnelle négative qui détermine les individu.e.s qui la subissent à être constamment à l'affût, à l'écoute du monde et des autres [...] pour nier, minimiser, désamorcer, encaisser, amoindrir ou éviter la violence, pour se mettre à l'abri, pour se protéger, pour se défendre. Il s'agit alors de développer une série de raisonnements pour déchiffrer autrui, pour rendre raisonnable, «normal» son action, à déployer des gestes, des attitudes, des actions pour ne pas l'"énerver » [.] Il n'est plus question ici de se "soucier des autres" pour faire quelque chose qui les aide, les soigne, les réconforte, les rassure, les sécurise, mais bien de se soucier des autres pour anticiper ce qu'ils veulent, vont ou peuvent faire de nous ${ }^{27}$.

Dès lors, Dorlin avance l'idée que l'attention et le souci des autres ne résultent pas d'un lien affectif positif, mais, au contraire, d'un ensemble de contraintes systémiques, historiques et politiques. Les femmes et les groupes minoritaires ont eu à développer une capacité d'attention beaucoup plus forte, car leurs statuts de subalternes impliquent une attention forcenée (car forcée par les rapports de domination) aux autres. Ici, le souci des autres est surtout compris comme un dispositif de survie propre aux subjectivités précarisées et donc, habitées par le fait d'être constamment en éveil et sur ses gardes. La perception de la souffrance, l'attention et le fragile souci des autres perdent leur caractère positif et moral pour être saisis à partir des effets de la domination et de la structure du pouvoir sur les subjectivités.

25. Ibid., p. II 5 .

26. Elsa Dorlin, Se défendre. Une philosophie de la violence, Paris, La Découverte, 2017.

27. Ibid., p. 175. 
Ferrarese, tout comme Dorlin, examine la compartimentation du geste moral et son assignation à des catégories précises. Cependant, dans sa reprise d'Adorno, Ferrarese déploie une forte critique de l'idée de "conservation de soi » : si l'attention aux autres résultait d'une réaction de survie, alors l'individu froid ne diffèrerait en rien de la personne qui prend soin des autres, puisqu'il s'agirait simplement de deux modes différents de conservation de soi.

Ferrarese semble donc porter une sympathie envers les éthiciennes du care et défendre une conception de l'attention et du souci des autres en tant qu'ils constituent des éléments moraux essentiels aux maintiens de vies viables et à la perpétuation du monde. Dans cette optique, l'attention et le souci des autres relèvent à la fois d'une compartimentation sociale (injuste et même violente) et d'une capacité à voir la personne singulière, les petits riens banals, car trop quotidiens, ou encore les choses qu'on ne veut pas voir pour éviter de s'en occuper. L'attention suppose donc une capacité à percevoir ce qui, socialement, n'a pas de visibilité ou très peu d'importance. En tant qu'il porte une attention à ce qui n'est pas prestigieux, le souci des autres semble alors faire écho à la prescription de la morale minime chez Adorno, soit de résister à ce que le monde extérieur fait de nous, notamment à la tendance à l'efficacité et à l'instrumentalisation des relations humaines. Se soucier des autres dans un monde indifférent est une forme de résistance à l'indifférence, même si ce souci est par ailleurs modelé socialement. Le care — et c'est là peut-être un nœud central - fonctionnerait donc à l'intérieur du règne de la froideur, il est même administré en partie par lui, mais en même temps, quelque chose dans le care s'arracherait à la froideur. En ce sens, Ferrarese est à mi-chemin de l'approche de Dorlin: si elle admet l'aspect socialement construit de la fragilité du souci des autres, il y a, dans l'acte même du souci pour autrui, une forme de moralité qui s'arrache des relations de pouvoir pour constituer autre chose. On a l'impression que le souci pour autrui se situe dans une espèce de mise entre parenthèses phénoménologique dans laquelle la perception de la relationnalité humaine est possible: quelqu'un perçoit à un moment quelqu'un d'autre qui souffre et répond en tentant de diminuer sa souffrance.

\section{La question de la souffrance}

J'ai mentionné la répulsion face à l'horreur de la souffrance d'autrui élaborée par la lecture adornienne de Ferrarese. Après avoir examiné la compartimentation de l'attention et du souci pour les autres déférées à des catégories de population souvent dominées, Ferrarese aborde les subjectivités impuissantes chez Adorno. Au contraire des subjectivités froides, les subjectivités impuissantes ressentent davantage l'impulsion face à l'horreur de la souffrance d'autrui. Ces subjectivités sont marquées par la fêlure, au sens où elles n'incarnent pas aussi bien l'idéal de conservation de soi: elles sont pleines de fissures, par lesquelles elles sont affectées de la souffrance 
d'autrui, et c'est pour cela qu'elles perçoivent ce à quoi les subjectivités froides sont généralement indifférentes.

Dès lors, on pourrait se demander quelles sont les conditions matérielles de cette impulsion. Relèvent-elles surtout de la distribution des rôles sociaux et genrés - de la compartimentation des relations? Mais alors, comment décompartimenter le souci des autres?

Il me semble que nous pourrions trouver des éléments de réponse dans les écrits de Simone Weil28. Plusieurs théoriciennes du care réfèrent à Weil afin de creuser le phénomène de l'attention dans une perspective générale et critique $^{29}$. Si Weil ne s'est pas elle-même définie comme féministe, il importe de souligner que, contrairement à Adorno, cette dernière n'a jamais tenu des propos caricaturaux sur les femmes, ce qui facilite par ailleurs la transition du domaine précis qu'est la littérature sur l'éthique du care vers une prise en considération plus générale de ce que le capitalisme fait des relations humaines. D'autant que la pensée de Weil ne bénéficie pas de l'immense visibilité de celle d'Adorno ${ }^{30}$. Ainsi, je terminerai mon commentaire en ouvrant sur la perspective weilienne de l'attention, considérant qu'elle donne des pistes pour réfléchir à la tension qui touche la source du souci des autres, qui relèverait soit d'un conditionnement propre à la catégorisation sociale, soit d'une capacité qu'on peut développer et qui permet de voir réellement autrui en dehors des normes de reconnaissabilité.

28. Née à Paris en I909 dans une famille juive non pratiquante, Weil est formée dans les meilleures institutions françaises et rapidement remarquée pour ses grandes qualités philosophiques. Pourtant, dès I935, à l'âge de 25 ans, elle décide de quitter l'enseignement et d'aller travailler en usine chez Renault afin de comprendre l'oppression ouvrière. Elle part ensuite à Barcelone dans la colone Durutti afin de combattre auprès des anarchistes. Blessée (parce qu'elle a marché dans une marmite d'huile bouillante), elle rentre à Paris dégoûtée par les conséquences de la guerre sur le jugement humain. À partir de 1936, Weil fait une expérience mystique et s'intéresse de plus en plus au christianisme et aux religions en général. Elle refuse le baptême et demeure critique de l'institution catholique. Weil part en Italie et en Allemagne tenter de comprendre le surgissement du fascisme et du nazisme, puis participe à la résistance à Marseille. Elle meurt alors qu'elle travaille au sein de la Résistance à Londres avec De Gaulle, en I943, à l'âge de 34 ans. On retient d'elle (entre autres) ses écrits sur la guerre et la domination, sur le travail et l'usine, sur les religions, sur les droits et sur les conditions matérielles d'une société juste. Sa pensée s'ancre dans une forme de matérialisme critique: pour Weil comme pour Adorno, la souffrance et les contradictions sont des éléments révélateurs que la théorie doit prendre en considération.

29. Voir notamment les travaux de Sophie Bourgault: "Attentive Listening and Care in a Neoliberal Era: Weilian Insights for Hurried Times ", Etica \& Politica / Ethics \& Politics, Volume XVIII, n 30 , 20I6, p. 3II-339 ainsi que "Beyond the Saint and the Red Virgin: Simone Weil as Feminist Theorist of Care ", Frontiers: Journal of Women's Studies, Volume 35 , $\mathrm{n}^{\circ} 2,20 \mathrm{I} 4$, p. I-27).

30. Sur ce point, il me semble qu'on réfère encore trop peu, dans l'histoire des idées, à des philosophes femmes pour réfléchir à l'aspect général du lien humain et des rapports de pouvoir. 
Simone Weil, dans son expérience comme ouvrière en usine, a analysé l'oppression tayloriste. Elle observe dans le travail sur machine une réduction de l'attention à sa plus faible expression, qui est proportionnelle à la réduction de l'expérience du monde. À l'inverse, on pourrait dire que le travail en régime néolibéral se déploie surtout depuis une certaine valorisation, voire parfois une exigence, du régime de l'attention faible, l'attention multitask, qui est une autre manière de détourner la subjectivité d'une attention véritable à autrui ${ }^{31}$. Selon Weil, l'oppression au travail, la peur, le désir d'accumuler, l'envie d'être le meilleur contribuent à l'indifférence envers les autres. Comme le mentionne Weil, en usine:

[u]ne force presque irrésistible, comparable à la pesanteur, empêche alors de sentir la présence d'autres êtres humains qui peinent eux aussi tout près; il est presque impossible de ne pas devenir indifférent et brutal comme le système dans lequel on est pris; et réciproquement la brutalité du système est reflétée et rendue sensible par les gestes, les regards, les paroles de ceux qu'on a autour de soi ${ }^{32}$.

L'exercice de la perception serait ainsi un premier pas vers la capacité, possible pour tous et toutes, d'être sensible à la souffrance des autres, perception néanmoins suspendue à certaines conditions matérielles (par exemple avoir une certaine liberté de temps, des moments de silence, la possibilité de se sentir en sécurité, etc. ${ }^{33}$. Seulement, Weil considère que la souffrance réelle est souvent inaudible, car ce sont les plus forts qui sont les plus entendus. Dès lors, la perception, l'attention et le souci des autres impliquent aussi un aspect matériel, un rapport important au corps, puisque les mots et les discours sont accaparés par les plus puissants. Les personnes malheureuses vivent souvent une injustice herméneutique: elles n'ont pas les mots pour décrire leur situation vécue. C'est en ce sens que

la pensée de la souffrance n'est pas discursive. À la douleur physique, au malheur, la pensée se heurte comme la mouche au verre, sans pouvoir progresser aucunement ni y découvrir rien de nouveau, et sans pouvoir s'empêcher d'y revenir. Ainsi s'exerce et se développe la faculté d'intuition ${ }^{34}$.

L'apprentissage de l'attention s'inscrit donc dans une logique sensible. Il faut accepter de se taire, de ne plus penser à soi-même. En fait, l'attention véritable est un moment de détournement du soi: lorsque je porte attention à quelqu'un ou à quelque chose, au moment précis où je le fais, je ne pense pas à moi.

31. Pour creuser davantage la question du multitâche, voir Yves Citton, Pour une économie de l'attention, Paris, Seuil, $20 \mathrm{I} 4$.

32. Simone Weil, Euvres complètes II, Cahiers 2: Écrits historiques et politiques. L'expérience ouvrière et l'adieu à la révolution, Paris, Gallimard, I99I, p. 297.

33. Pour une liste des besoins humains, voir L'Enracinement dans Simone Weil, Euvres, Paris, Quarto, Gallimard, I999.

34. Simone Weil, Euvres complètes VI, 3 : Cahiers (février I942-juin I942), Paris, Gallimard, 2002, p. I82-I 83 . 
Cette idée rejoint en partie la critique de la conservation de soi adornienne. En effet, pour Weil, une partie du soi est le résultat des rapports de force et des prestiges sociaux. Il faut, pour être à l'écoute de quelqu'un qui ne compte pas socialement, détruire les prestiges qui, en nous, nous confortent et nous confirment. Cet exercice du souci de soi pour se soucier d'autrui est extrêmement difficile et exige de consentir à une forme de souffrance, puisqu'il faut s'arracher (et c'est un travail perpétuel) de ce que les rapports de domination ont déposé en nous. De plus, il faut être en mesure de recevoir altérité de l'autre auquel on porte attention, sans préconception et sans attente. La pensée doit être à vide. Cette double difficulté contribue au fait que, pour Weil, il est extrêmement rare que nous portions attention à la souffrance d'autrui.

Si la question d'un travail non servile préoccupe Weil, c'est que le travail actualise l'humanité dans sa possibilité perceptive, qui est aussi une perception non discursive et non orientée, mais inhérente à la matérialité du corps: bref, une perception pré-égoïque, vierge de l'empreinte du pouvoir. L'attention se développe au travers de notre sensibilité corporelle et psychique au monde. Elle est inhérente à la socialisation, à la matérialité et aux fonctionnements des institutions. Paradoxalement, l'apprentissage de l'attention se fait toujours à l'intérieur des conditions matérielles. C'est en creusant au plus profond du social jusqu'au point de s'en arracher que la subjectivité, pour un instant du moins, porte un regard attentif sur ceux et celles qui n'ont guère de visibilité, qui ne sont ni prestigieux ni dominants, qui sont parfois marginalisés ou sans-voix. Cet arrachement, on pourrait le concevoir comme une cessation (peut-être seulement momentanée) de l'injonction à s'auto-conserver, un moment de fissure qui favorise la capacité à être affecté. En ce sens, l'attention et le souci des autres sont un apprentissage, car plus on fait l'effort d'être attentif, plus le geste adéquat inhérent au discernement de l'évaluation morale est susceptible d'émerger, même dans un monde mauvais.

C'est vers cette direction que semble tendre le livre d'Estelle Ferrarese lorsqu'elle conclut avec Adorno sur le rôle du théoricien critique, lequel implique de se maintenir dans la « pourriture $^{35}$ ». Ferrarese réussit avec force à interroger le geste moral sans le désincarner de son contexte historique, d'une part, sans en faire non plus un pur conditionnement, d'autre part. Le dialogue riche et soutenu entre la pensée d'Adorno et les éthiques du care ouvre la voie à un désenclavement des théories de l'École de Francfort et des théories féministes qui s'avère prometteur et dont, je l'espère, jailliront de nouvelles recherches.

35. E. Ferrarese, La fragilité du souci des autres, op. cit., p. I42. Ce qui rejoint tout à fait la démarche weilienne, si l'on considère que la philosophe a préféré à maintes reprises partager les duretés des plus vulnérables de son temps, plutôt que de se satisfaire de l'enseignement. 